\title{
Closing -Loops Producing Added-Value Products as a Cost-Reduction Strategy in the Operation of Biorefineries
}

\author{
William Kerstein ${ }^{1}$, Elena Ten ${ }^{2}$, Luisa Dempere ${ }^{1,3}$, and Wilfred Vermerris ${ }^{4}$
}

1 Materials Science and Engineering, University of Florida, Gainesville, FL 32611

2 Agronomy Department, University of Florida, Gainesville, FL 32610

3 Major Analytical Instrumentation Center, University of Florida, Gainesville, FL 32611

4 Microbiology Department, University of Florida, Gainesville, FL 32610

Multiple studies are being conducted with the purpose of exploring the use of waste residues generated during the bioethanol processing of sugar cane bagasse as raw materials for addedvalue products, primarily Lignin. Lignin is next to cellulose the most abundant biopolymer on earth. Lignin coats cellulose micro-fibrils and other components in the cell wall preventing collapse and providing support to the cell walls in biomass. Being a renewable energy source, biomass is a common feedstock for biopolymers, paper and biofuel production. Lignin left over from the production of biofuels is a significant portion of biomass (up to $25 \%$ ), and it is often burned to generate heat for the distillation of alcoholic fuels from the fermentation liquor. With increasing demand of sustainable energy sources, we are exploring the recovery and modification of Lignin to reduce costs of biofuel production.

In this study we worked with pre-treated lignin provided by the University of Florida Ethanol Pilot Plant and The Stan Mayfield Biorefinery. Lignin is isolated from biomass using a two-step hydrolysis in phosphoric acid followed by enzymatic hydrolysis that removes polysaccharides. This process solubilizes approximately eighty to ninety percent of the hemicellulose, leaving the cellulose and lignin largely intact as polymers.

Our group produces lignin-based nanotubes using a sacrificial template of alumina membranes. Isolated Lignin is covalently linked to the inner walls of activated alumina membranes and then layers of dehydrogenation polymer are added onto this base layer via a peroxidase-catalyzed reaction. The thickness of the polymer layer deposited within the pores is selected by using phenolic monomers displaying different reactivity. Thus, we synthesize nanotubes with a wall thickness of approximately $15 \mathrm{~nm}$ and nanowires with a nominal diameter of $200 \mathrm{~nm}$. These novel nanostructures are flexible and can be bio-functionalized easily and specifically. Unfortunately, the cost of the alumina membranes is the limiting step in the upscaling of the synthesis of lignin nanotubes. Thus, the investigation of the generation of secondary products from the membranes processing waste was pursued as a strategy to reduce costs. Closed loops systems are based in the concept of "waste equals food" where by-products or waste of a specific process are used as raw or initial materials for other products. The waste solution generated in the dissolution of the membranes to release the nanotubes was investigated as a precursor mixture for the synthesis of Zeolites than can be used in some of the catalyzed stages of the bioethanol production. Figures 1 and 2 show the images of the synthesized Zeolites.

\section{References:}

[1] Robinson, H. (2011). AFI AlPO4-5. Verified syntheses of zeolithic materials. Elsevier. 


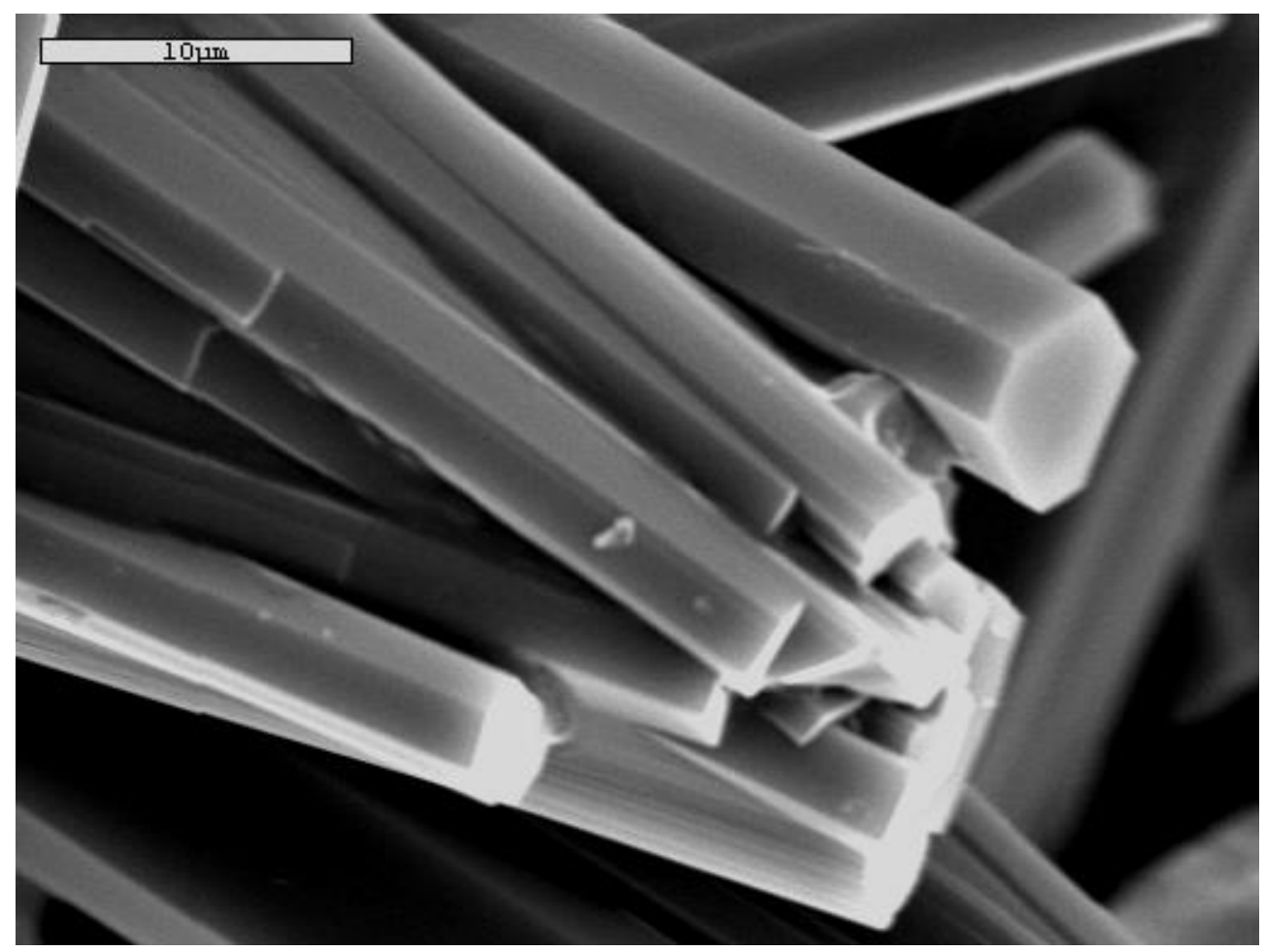

Fig. 1 Secondary electron image of Zeolite crystals; magnification 3,000X.

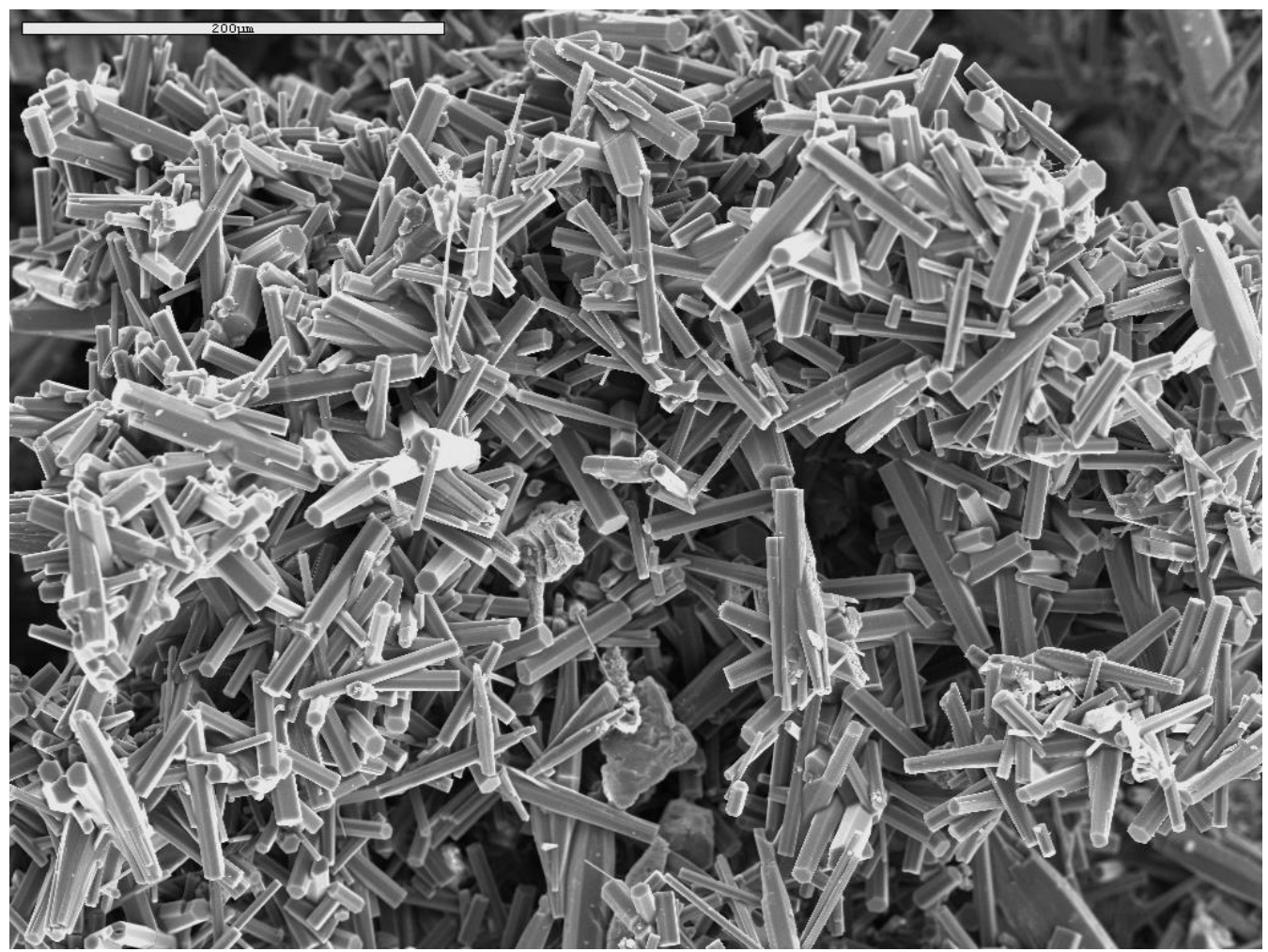

Fig. 2 Secondary electron image of Zeolite crystals; magnification 200X. 